\title{
The Incidence of Thyroid Cancer in England and Wales over A Ten Year Period
}

\section{Louise Marie Evans, ${ }^{1}$ Michael Stechman, ${ }^{2}$ David Owens ${ }^{2}$}

\section{Introduction}

\section{$\underline{\text { ABSTRACT }}$}

The incidence of thyroid cancer has increased worldwide, whether a real or apparent increase is debated. Literature from the USA suggests greater diagnostic scrutiny, environmental and genetic factors may all play a part. This increase will result in a greater number of referrals for surgical assessment. This study examined the trend in incidence of thyroid cancer in England and Wales.

\section{Materials and Methods}

A retrospective study, using the HES database over the period 2000-2010. Data were extracted of all newly diagnosed thyroid cancers in England and Wales and the age at diagnosis. Data were examined for the change in incidence of thyroid cancer diagnosis dependent on the age group of the patient using the linear regression model.

$\underline{\text { Results }}$

45411 patients were identified. In England the incidence of thyroid cancer rose from 5.7/100,000 of the population in 2000 to 9.9/100,000 in 2010 and in Wales it rose from 3.5/100,000 in 2000 to 7.5/100,000. There was a statistical increase (P $\leq 0.02)$ $(t$-stat $>2)$ in the diagnosis of thyroid cancers across all age groups with exception of the 0-14 age group $(P>0.5)$.

\section{Conclusion}

There has been a statistical increase in the incidence of thyroid cancer. This is likely to impact on hospitals and cancer service resources. An increase in surgical demand and the coinciding ageing population highlights the importance of further investigation into the etiology, use of imaging, patient demographics, histology and overall mortality of this patient group. Kevwords

Thyroid Neoplasms; Incidence; England; Wales

$\mathrm{T}$ The incidence in thyroid cancer has increased worldwide, with countries such as Scotland, ${ }^{1}$ the $\mathrm{USA}^{2}$ and $\mathrm{Mexico}^{3}$ all reporting an increase. Whether this is a true or apparent increase is debated.

The effect of radiation from atmospheric testing of nuclear weapons in the 1950-60s and female reproductive patterns have been considered causative factors for the rise. ${ }^{4,5,6}$ The more favored view however is that improvements in technology, more sophisticated imaging techniques and a greater accessibility to

1 - Morriston Hospital, Swansea, Wales, United Kingdom

2 - University Hospital of Wales, Cardiff, United Kingdom

\section{Corresponding author:}

Louise Marie Evans

email: louiseevans 5@hotmail.co.uk ultrasound scanning has resulted in diagnosis of thyroid cancers which may have otherwise gone unnoticed.

There has been little written about the impact of this increase on the patients, the doctors' workload and the health service as a whole. With this in mind we investigated the trends in the incidence of thyroid cancer in England and Wales over a 10-year period.

\section{Materials and Methods}

A retrospective analysis of Health Solutions Wales (PEDW) and the English Hospital Episode Statistics (HES) annual data was conducted over a ten-year period, from the year 2000 to 2010. The numbers of patients who were newly diagnosed with thyroid cancers in England 
and Wales adjusted for 100,000 of population each year were examined. The incidence of thyroid cancer rates within four different age groups, 0-15, 16-59, 60-74 and $\geq 75$ years for both England and Wales were analysed. Statistical analysis for change over time was examined using a linear regression model (Microsoft Excel, Microsoft, USA). $\mathrm{P}<0.05$ was accepted as statistically significant.

\section{Results}

Between 2000 and 2010, 45411 patients were newly diagnosed with thyroid cancer in England and Wales representing $0.08 \%$ of the population. Thyroid cancer was found to be most common in the $\geq 60$ age group.

Overall, in England, the incidence of thyroid cancer increased significantly from $5.7 / 100,000$ of population in 2000 to $9.9 / 100,000$ of population in 2010. In Wales the incidence rose from $3.5 / 100,000$ in 2000 to 7.5/100,000 (Fig. 1).

The incidence of thyroid cancer in the 4 age-bands collated by HES and PEDW exhibited some variation. In patients aged $0-14$ years, there was no significant change over time and the incidence remained between 0 and 0.1 per 100,000 of population over the study period

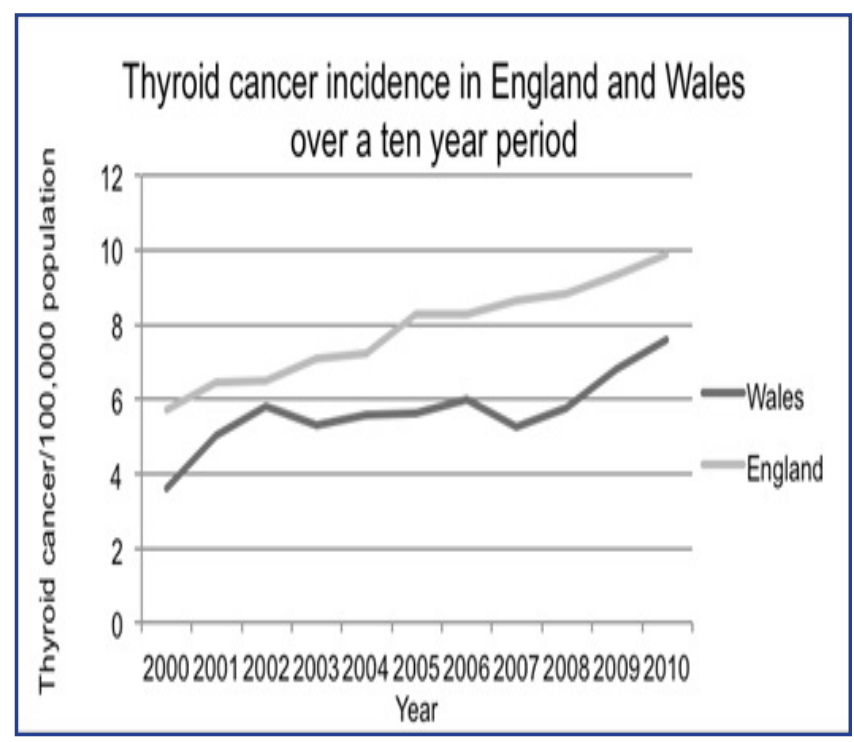

Fig.1. Incidence of Thyroid Cancer

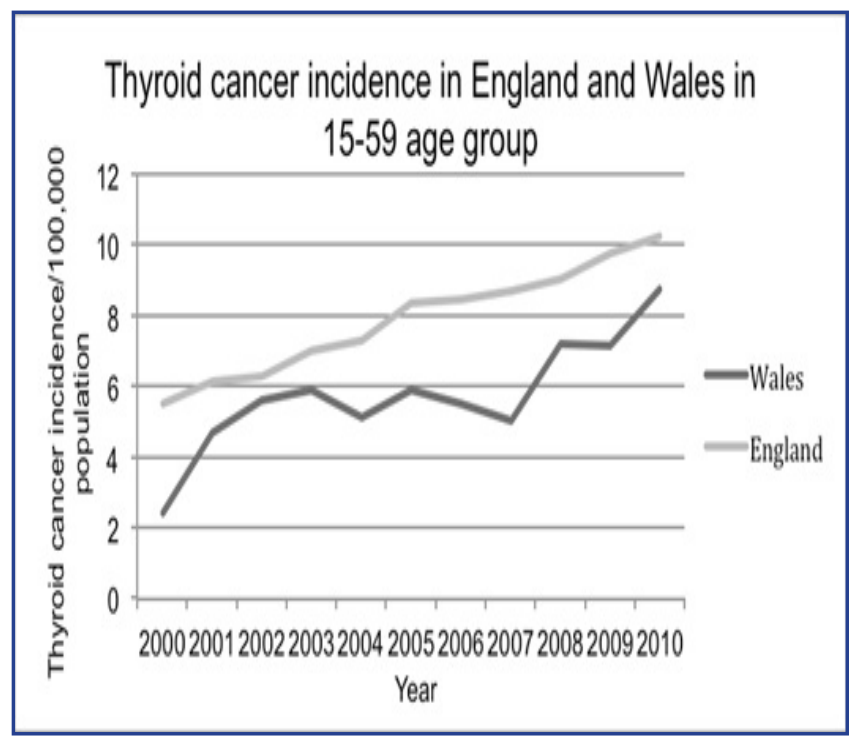

Fig.2. Incidence of Thyroid Cancer in 15-59 years age group

$(\mathrm{P}=0.6)$. In all other age groups (15-59 years, $60-74$ years, and 75 years and above) there was a significant increase $(\mathrm{P} \leq 0.02)(\mathrm{t}-\mathrm{stat}>2)$ in the incidence of thyroid cancer from 2000 to 2010.

The changes in incidence over time are shown in figures 2, 3 and 4.

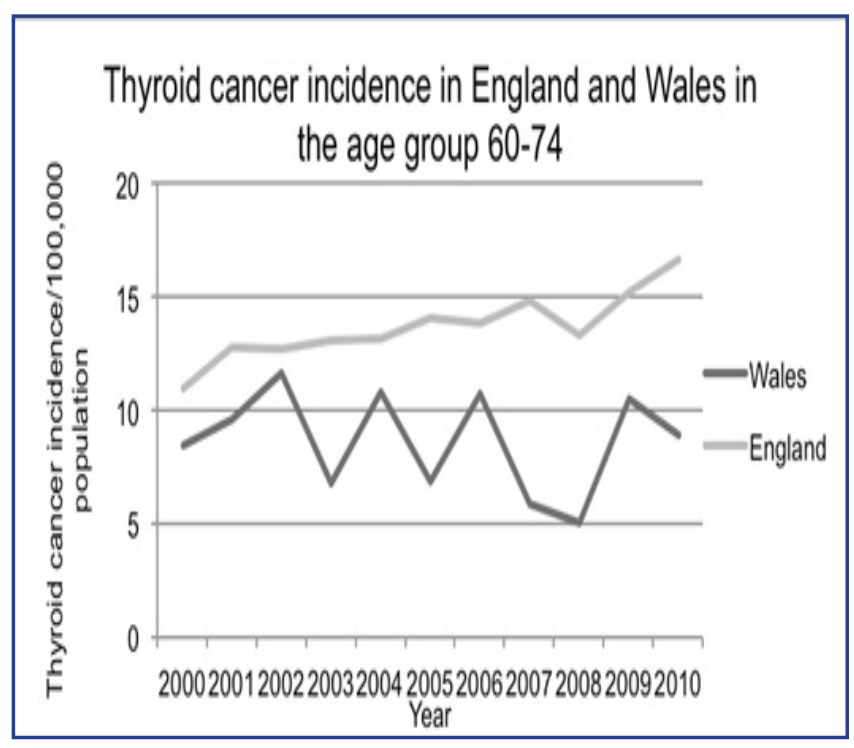

Fig.3. Incidence of Thyroid Cancer in 60-74 years age group 


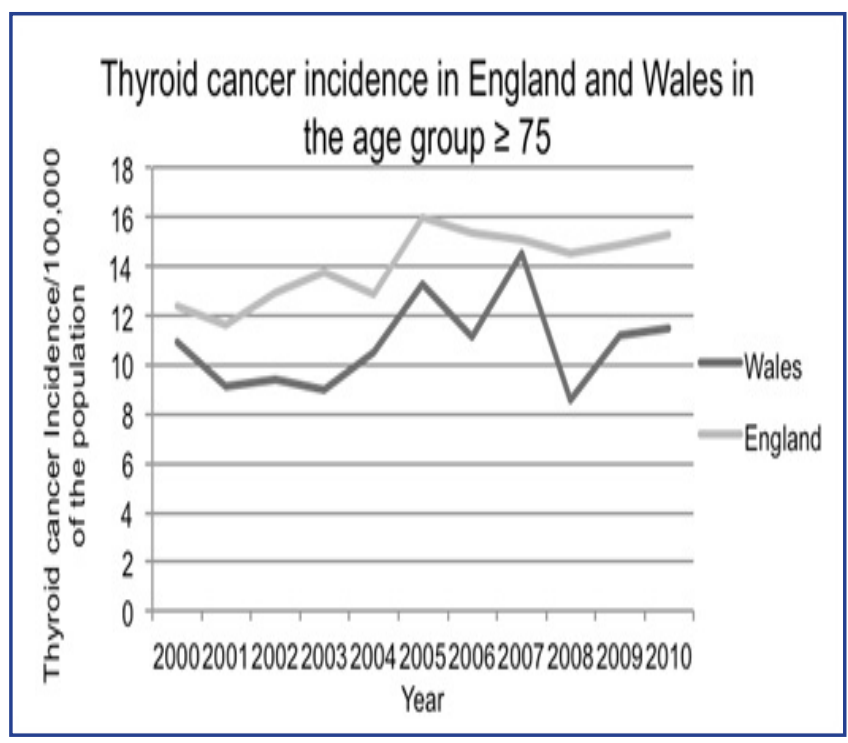

Fig.4. Incidence of Thyroid Cancer in $>75$ years age group

\section{Discussion}

These data demonstrate that there has been a significant and progressive increase in the incidence of thyroid cancer in adult patients from 2000-2010. The greatest increase was observed in those aged $\geq 60$. However the mortality rate in England has remained relatively static. ${ }^{7}$

There have been previous comments on the validity of HES and PEDW data, which is frequently entered by non-medically trained personnel. However it has been demonstrated in systematic reviews that there is a good correlation between reported and actual validated data, with an estimated coding accuracy of 88 to $92 \%{ }^{8,9}$

Although this study does not directly address the underlying reasons for this increase in thyroid cancer incidence it is important to consider all factors. Radiation is an established risk factor; nuclear weapons testing in the 50's and 60's and the Chernobyl disaster in the 1980's make a good argument for the possibility that radiation is to account. However some who have considered the geographical distribution of thyroid cancers have felt that the areas with a high incidence of disease is inconsistent with exposure to radioactive fallout and that this makes radiation an unlikely major causative factor. ${ }^{6,10}$

The influence of female hormones and reproductive factors on the thyroid has also been debated due to the high proportion of young females that develop thyroid disease. One report suggests that women who had irregular menstruation and women who have undergone a bilateral oophorectomy were at greater disease risk. ${ }^{11}$ However many reports are inconclusive or show no link between the two, with calls for further research to be completed on the subject. ${ }^{4,5}$

Another possible explanation is that the widespread accessibility and use of imaging has lead to an apparent increase in thyroid cancer numbers. Of the cancers newly diagnosed it is noted that there has been an increase in the number of small papillary thyroid cancers. ${ }^{1,2,3}$ This coinciding increase $\mathrm{e}^{2,7}$ may lead us to suggest that we are detecting a greater number of patients who may have subclinical disease. Higher incidences of thyroid disease has also been seen in patients of a higher socioeconomic status and in patients with health insurance cover. ${ }^{12,13}$ As a result these patients will have increased access to imaging. ${ }^{12}$

The management of small thyroid tumors poses a number of difficulties with regards to diagnosis and management of the patient. ${ }^{14}$ Some small tumors that are asymptomatic remain dormant in the general population and do not manifest. Ultrasound fine needle aspiration biopsy permits the identification of cancers smaller than $1 \mathrm{~cm} .{ }^{14}$ Although it is beneficial to catch disease at an early stage the implications of how we discuss the risks and benefits of surgery and anesthesia in the context of the natural progression of the disease should be considered. The cost implication and the capacity for long term follow up also need to be addressed. This is especially important in the context of an aging population often with a range of other comorbidities. ${ }^{15}$ The incidence of thyroid cancer increases with age. ${ }^{15}$ Our data has shown the increase in incidence in the over 65 age groups to be the greatest, despite the fact that papillary cancers present more commonly in the 30-50 age group. ${ }^{15}$ With a population that has aged significantly during the last two decades and that is predicted to increase further during the next two decades, ${ }^{16}$ this may present further challenges in managing these patients. 


\section{Conclusion}

These data confirm that there has been a significant increase in thyroid cancers in adult patients over the last decade (2000-2010). The reasons for this are likely to be a result of a greater diagnostic scrutiny but may also be multi-factorial. The long-term trend and effects of this increasing intervention remains to be seen. An increase in surgical demand and the coinciding ageing population highlights the importance of further investigation into the etiology, use of imaging, patient demographics, histology and overall mortality of this patient group.

\section{References}

1. Reynolds RM, Weir J, Stockton DL et al. Changing trends in incidence and mortality of thyroid cancer in Scotland. Clin Endocrinol (Oxf). 2005; 62(2): 156-62

2. Davies L, Welsh HG. Increasing incidence of thyroid cancer in the United States, 1973-2002. JAMA 2006; 295(18): 2164-7

3. Albores-Saavedra J, Henson DE, Glazer E et al. Changing patterns in the incidence and survival of thyroid cancer with follicular phenotype--papillary, follicular, and anaplastic: a morphological and epidemiological study. Endocr Pathol. 2007 Spring; 18(1): 1-7

4. Levi F, Franceschi S, Gulie C et al. Female thyroid cancer: the role of reproductive and hormonal factors in Switzerland. Oncology 1993; 50(4): 309-15

5. Franceschi S, Fassina A, Talamini R et al. The influence of reproductive and hormonal factors on thyroid cancer in women.
Rev Epidemiol Sante Publique 1990; 38(1): 27-34

6. dos Santos Silva I, Swerdlow AJ. Thyroid cancer epidemiology in England and Wales: time trends and geographical distribution. Br J Cancer 1993; 67(2): 330-40

7. Oxford Cancer Intelligence Unit. Profile of Head and Neck Cancers in England. Incidence, Mortality and Survival.

8. Campbell SE, Campbell MK, Grimshaw JM et al. A systematic review of discharge coding accuracy. J Public Health 2001; 23: 205-11

9. Burns, EM, Rigby E, Mamidanna R, et al. Systematic review of discharge coding accuracy. J Public Health 201234 (1): 138-48

10. Amphlett B, Lawson Z, Abdulrahman GO Jr et al. Recent Trends in the Incidence, Geographical Distribution, and Survival From Thyroid Cancer in Wales, 1985-2010. Thyroid 2013 June

11. Mack WJ, Preston-Martin S, Bernstein L et al. Reproductive and Hormonal Risk Factors for Thyroid Cancer in Los Angeles County Females. Cancer Epidemiol Biomarkers Prev. 1999; 8(11):991-7

12. Guay B, Johnson-Obaseki SE, McDonald JT et al. Incidence of Differentiated Thyroid Cancer by Socioeconomic Status and Urban residence: Canada 1991-2006. Thyroid. 2013 Sep 4

13. Siu S, McDonald JT, Rajaraman M et al. Is lower socioeconomic status associated with more advanced thyroid cancer stage at presentation? A study in two Canadian centers.Thyroid. 2013 Sep 10

14. Ernest L. Mazzaferri, MD. Managing Small Thyroid Cancers. JAMA 2006; 295(18): 2179-82

15. Boltz MM, Hollenbeak CS, Schaefer E et al. Attributable costs of differentiated thyroid cancer in the elderly Medicare population. Surgery. 2013 Aug 22. Pii: S0039-6060 (13) 00364-4.

16. Office for National Statistics. March 2012. Population ageing in the United Kingdom, its Constituent Countries and the European Union. http://www.ons.gov.uk/ons/dep171776_258607.pdf 